\title{
Letter to the Editor concerning: "Results of pectus excavatum correction using a minimally invasive approach with subxyphoid incision and three-point fixation"
}

\author{
Joseph Sujka ${ }^{1}$ Shawn St. Peter ${ }^{1} \cdot$ Tolulope A. Oyetunji $^{1}$
}

Accepted: 6 April 2018 / Published online: 11 April 2018

(c) Springer-Verlag GmbH Germany, part of Springer Nature 2018

\section{Dear Editor,}

We read with great interest the article by Bond et al. regarding results of pectus excavatum correction using a modified minimally invasive approach with subxyphoid incision and three-point fixation [1]. The authors demonstrated safety and excellent outcomes with the procedure comparable to the standard Nuss procedure. Interestingly, since 1999, our institution modified the minimally invasive Nuss procedure to include a subxyphoid incision, as described by the author. However, we utilize a two-point fixation. Our institution recently reviewed our experience with the modified Nuss procedure. In our analysis of 554 patients over a 15 -year period, our rate of bar repositioning for rotation was $<1 \%$ and our rate of stabilizer removal due to chronic discomfort was similarly low at $1.4 \%$ [2]. We have also not encountered any cardiac injury or required multiple bars. We have occasionally used a single stabilizer with no increase in the incidence of bar displacement in this cohort (unpublished data).

The authors in their article describe a three-point fixation compared to the standard two-point fixation. The cited rate of bar displacement in their study was $2.7 \%$. As mentioned earlier, our rate of bar displacement with the two-point fixation was $1.4 \%$, lower than that in this study. The inclusion of a third fixation point as described by the author, therefore, may be unnecessary and possibly eliminated to further simplify the steps of the procedure during placement. More

so, opening the third (subxyphoid) incision may be required during bar removal, if the fixation has to be released. This will potentially not be required with a two-point fixation.

We again compliment the authors on their excellent article and thank them for their contribution to it.

Funding No funding was received to conduct this study.

\section{Compliance with ethical standards}

Conflict of interest The authors have no conflict of interest to disclose.

Ethical approval This article does not contain any studies with human participants or animals performed by any of the authors.

Informed consent Informed consent was waived by our IRB due to the fact that the data collected for this study were retrospective and de-identified.

\section{References}

1. Bond SJ, Rapstine E, Bond JM (2018) Results of pectus excavatum correction using a minimally invasive approach with subxyphoid incision and three-point fixation. Pediatr Surg Int 34:75-78. https ://doi.org/10.1007/s00383-017-4195-z

2. Gould J, Sharp R, Peter S et al (2016) The minimally invasive repair of pectus excavatum using a subxiphoid incision. Eur $\mathrm{J}$ Pediatr Surg 27:002-006. https://doi.org/10.1055/s-0036-15875 85

This comment refers to the article available at https://doi. org/10.1007/s00383-017-4195-z.

Tolulope A. Oyetunji

taoyetunji@cmh.edu

1 Department of Surgery, Children's Mercy Hospital,

Kansas City, MO 64108, USA 